\title{
The proliferation marker thymidine kinase 1 in clinical use (Review)
}

\author{
JI ZHOU, ELLEN HE and SVEN SKOG
}

Sino-Swed Molecular Bio-Medicine Research Institute, Shenzhen 518057, P.R. China

Received May 16, 2012; Accepted August 20, 2012

DOI: $10.3892 / \mathrm{mco} .2012 .19$

\begin{abstract}
Tumor-related biomarkers are used for the diagnosis, prognosis and monitoring of treatments and follow-up of cancer patients, although only a few are fully accepted for the detection of invisible/visible tumors in health screening. Thymidine kinase 1 (TK1), a cell cycle-dependent and thus a proliferation-related marker, has been extensively studied during the last decades, using both biochemical and immunological techniques. Therefore, TK1 is an emerging potential proliferating biomarker in oncology that may be used for the prognosis and monitoring of tumor therapy, relapse and survival. In addition, TK1 concentration in serum (STK1p) is a useful biomarker in healthy screening for the detection of potential malignancy development as well as the identification of early-stage tumors, with a few false-positive cases (ROC value, 0.96; tumor proliferation sensitivity, 0.80; specificity, 0.99). In this review, we examine results regarding the expression of STK1p and TK1 in relation to cancer patients and STK1p in health screening published between 2000 and 2012. The use of tumor-related markers recommended by international cancer organizations is also discussed. This review provides valuable information for applications in tumor patients, in health screening and for cancer research.
\end{abstract}

\section{Contents}

1. Introduction

2. Tumor-related tailored biomarker

3. Thymidine kinase 1

Correspondence to: Professor Sven Skog or Ji Zhou, Sino-Swed Molecular Bio-Medicine Research Institute, 2-304 Bio-tech Industry Incubator, High-tech Industrial Park, Gaoxin C, Ave. 1st, Shenzhen 518057, P.R. China

E-mail: svenisak@hotmail.com

E-mail: kevin.zhou@sstkbiotech.com

Key words: thymidine kinase 1, serum, immunohistochemistry, health screening, oncology, tumor marker, cell proliferation marker, concentration of thymidine kinase 1 in serum, activity of thymidine kinase 1 in serum, prognosis, monitoring tumor therapy, relapse, follow-up, survival
4. Thymidine kinase 1 in health care applications

5. Conclusion

\section{Introduction}

Cancer is a disease of abnormal proliferating cells. Mutations in certain enzymes and proteins associated with cell growth regulation leads to uncontrolled proliferation and thus malignancy. Non-invasive serological methods provide information of invisible tumors (early tumor detection) prior to detection by modern imaging, thus increasing the potential to cure patients. Furthermore, prognostic and predictive factors are crucial for identifying patients at a higher risk of relapse and for selecting the most appropriate systemic treatment for individual patients.

Several potential biomarkers are available at present and each marker has its own clinical characteristics. Thymidine kinase 1 activity in serum (STKa) is a cell-proliferating marker that has been used for the prognosis and monitoring of treatment, follow-up and survival in patients with lymphoma and leukaemia since 1983 (1). Similarly, subsequent to the development of anti-thymidine kinase 1 (TK1) antibodies by the Swed-Sino research group (2), TK1 concentration in serum (STK1p) became a useful tool for the prognosis, monitoring of tumor treatments, relapse, follow-up and survival, particularly in solid tumors. These results have been partly shown in recent reviews (3-7). STK1p was found to be an emerging potential cell proliferation biomarker for the early detection of premalignant and malignant diseases in health screening. This review examined results regarding the expression of STK1p and TK1 in tumor tissues (immunohistochemistry) and STK1p in health screening published between 2000 and 2012. The use of tumor-related markers recommended by international cancer organizations was also evaluated in the present review.

\section{Tumor-related tailored biomarker}

Biomarkers in cancer patients are divided into growth- and tumor-related markers. Growth-related markers are compounds involved in the regulation of growth and anti-growth signals, cell cycle, apoptosis, angiogenesis, adherence, tissue invasion and metastasis (3-10). At present, biomarkers, such as Ki-67, TK1, p53 and M30 are in commercial use in the clinic. The tumor-related types of biomarkers are not correlated with growth directly, but are present or absent in malignant cells, as 
reflected by their serum concentrations and tumor tissue expression. Examples of such biomarkers are carcinoembryonic antigen (CEA), cancer antigen 15-3 (CA15-3), CA19-9 and CA-125, reflecting epidermal cell carcinoma, neuron-specific enolase (NSE) and squamous (SCC)-Ag for the detection of squamous cell carcinoma and prostate-specific antigen (PSA) in prostate carcinoma. Guidelines for the use of these markers in the clinic have been published by the American Society of Clinical Oncology (ASCO), the American Cancer Society (ACS), the National Cancer Institute (NCI) and the National Comprehensive Cancer Network (NCCN) (Table I). An overview by Cigna (11) indicated that most tumor biomarkers are often insufficient to diagnose malignancies by themselves for the following reasons: i) the tumor-related biomarker level may be elevated in individuals with benign conditions, or not elevated in individuals with malignancies, particularly at the early stages of tumor growth; ii) most of the tumor-related biomarkers are not specific to a particular type of tumor; and iii) the tumor-related biomarker level can be elevated by more than 1 type of tumor. However, certain tumor-related biomarkers reflect stages and/or tumor grades and are therefore useful in predicting treatment response and recurrence, but are still not recommended for the screening of cancer diseases (11).

\section{Thymidine kinase 1}

TK1 is a cell cycle-dependent enzyme of the pyrimidine salvage pathway, catalyzing the phosphorylation of thymidine to thymidine monophosphate. TK1 concentration in the cell is low in the G1 phase of the cell cycle, increases during the $\mathrm{S} / \mathrm{G} 2$ phases and decreases in the late $\mathrm{G} 2 / \mathrm{M}$ phase. TK1 is low or absent in non-proliferating cells, with some exceptions. Transcriptional and translational mechanisms control the expression of TK1 (1-7,12-14). The degradation of TK1 in the $\mathrm{M}$ phase is due to ubiquitin-proteasome-related mechanisms (13), singling out TK1 as a useful biomarker for cell proliferation, and thus for malignancy. Using the thymidine analogue $\left[{ }^{125} \mathrm{I}\right]$-5-iodo-2-deoxyuridine as a substrate, a STKa assay was established for human tumors in the early 1980s (1). The STKa assay is useful for the prognosis of survival and the monitoring of tumor treatment mainly in patients with leukaemia, as well as Hodgkin's and non-Hodgkin's lymphoma (3-5)

In 2002, Sino-Swed TongKang Biological Technology Inc. (SSTK Inc., Shenzhen, China) developed chicken polyclonal anti-TK1 antibodies for serum test and mouse monoclonal anti-TK1 antibodies for immunohistochemistry, under the guidance of the Swedish TK1 Research Group. A highly sensitive and specific serological assay system based on an immune-enhancing chemiluminescent dot blot detection system (ECL dot blot), as well as a TK1 immunohistochemical method, were established in 2003 (2). The new immunological TK1 assay determined the concentration of STK1p instead of STKa, and was found to be useful both in patients with solid tumors and those with leukaemia and lymphoma (4-7,15-20). In addition, the commercial serum TK1 kit (SSTK Inc.) is useful for the detection of pre-malignancies and malignancies in health screening (21-23). Until May 2012, over 450,000 individuals participating in health screenings were tested for STK1p, of which data from 45,682 cases have been analysed and published (21-27). STK1p has also been determined in
6,124 diagnosed cancer patients with 15 types of malignancies (28-59). TK1 expression in tumor tissues (immunohistochemistry) has been analysed in 1,357 patients with 8 different types of malignancies (60-71).

\section{Thymidine kinase 1 in health care applications}

STK1p in health screening. An effective tumor-screening test, such as the STK1p assay should detect malignancies, i.e. physiological conditions that increase the risk of developing a tumor in the future $(6,7,21-27)$ as early as possible. Patients with an elevated STK1p (risk cut-off value $>2.0 \mathrm{pM}$ ) showed a significantly higher frequency of obesity, fatty liver and HBV infections, as well as various types of moderate/ severe pre-malignancies (26), conditions that increase the risk of developing malignancies over the years. Additionally, a significantly higher (3- to 5-fold) risk of developing malignancies in the course of 6 years was found (26). This is the first study to report TK1 concentration in serum to be useful in health screening.

A detection system of serum markers using immunological tools in health screening requires antibodies of high sensitivity and specificity, with no cross-reactivity of human serum. The sensitivity and specificity is determined by the receiver operation characteristic (ROC) analysis. A ROC-value $>0.95$ and a likelihood (+) value $>8.0$ is recommended for a health screening test. According to a ROC analysis carried out on the STK1p test developed by SSTK Inc., statistically significant differences were detected between pre-operational pre-malignant/malignant patients $(n=720)$, and healthy individuals/ individuals with diseases other than pre-malignancies/malignancies $(n=4,103)(26)$. Since STK1p is not a tumor marker, but a proliferation biomarker, the control group should comprise healthy individuals and individuals with proliferational non-malignant diseases and physiological changes affecting the STK1p level. The ROC value was found to be 0.96 and the likelihood (+) value 199.7. At a STK1p risk cut-off value of $2.0 \mathrm{pM}$, the sensitivity and specificity indices were 0.80 and 0.99 , respectively (26), showing that the STK1p serum assay is a highly reliable test, with a low number of false-positive cases (1/300). The STK1p values of healthy individuals with no known pre-malignancies/malignancies in the ECL dot blot assay were $0.4-0.8 \mathrm{pM}$.

The first STK1p preliminary test in health screening was conducted at the 301st Hospital in Beijing, China, during 20062007. This study included 72 individuals of middle age with no symptoms of malignancies or other diseases. Three individuals were found to have elevated STK1p values $(1.8,7.0$ and $7.1 \mathrm{pM})$ compared to the healthy individuals $(0.5 \mathrm{pM})$. The pathology examination showed esophagus squamous cell carcinoma, gastric adenocarcinoma and small adeno/squamous carcinomas of the lung, respectively (22). Despite the small scale of the present study, the STK1p assay has the potential to be used for identifying individuals with malignancies in a group of healthy people. Based on these results, large-scale health screening studies were initiated in China [the Third Xiang Ya Hospital, ZhongNan University, Changzha $(23,26,27)$, the General Hospital of the Jilin Oilfield, Jilin (24) and the Fujin People's Hospital, Fuzu (25)], the results of which also demonstrated that STK1p had the potential to identify individuals at 
Table I. Markers recommended for clinical applications by the cancer organizations ASCO, ACS, NCI and NCCN.

\begin{tabular}{llcccc}
\hline Markers & Malignancy & Diagnosis & Prognosis & Monitoring & Screening \\
\hline AFP & Liver & Yes & Yes & Yes & Yes \\
AFP + b-hCG & Testis, ovary & Yes & - & Yes & - \\
Calcitonin & Thyroid & Yes & - & - & - \\
CA 19-9 & Gastric, pancreatic & - & - & Yes & - \\
CA-125 & Ovary & - & - & Yes & - \\
CA-125 & Endometrial cancer & Yes & - & - & \\
CA-153, CA 27-29 & Breast & - & - & Yes & - \\
CEA & Colorectal & - & - & Yes & - \\
CEA & Liver & Yes & - & - & - \\
CgA & Neuroendocrine & Yes & - & - & - \\
Estrogen/progesterone receptor & Breast & - & - & Yes & - \\
HER-2/neu & Breast & - & - & Yes & - \\
hCG & Ovary, testis & - & - & Yes & - \\
NSE & Small lung cancer & - & Yes & Yes & - \\
PAP & Prostate & - & - & Yes & - \\
PSA & Prostate & - & - & Yes & - \\
Thyroglobulin & Thyroid & - & - & Yes & - \\
\hline
\end{tabular}

ASCO, American Society of Clinical Oncology; ACS, American Chemical Society; NCI, National Cancer Institute; NCCN, National Comprehensive Cancer Network; PSA, prostate-specific antigen; AFP, $\alpha$-fetoprotein; hCG, human chorionic gonadotropin; CA 19-9, cancer antigen 19-9; CA-125, cancer antigen 125; CA-153, cancer antigen-153; CA 27-29, cancer antigen 27-29; CEA, carcinoembryonic antigen; $\mathrm{CgA}$, chromogranin A; HER-2/neu, human epidermal growth factor receptor 2; NSE, neuron-specific enolase; PAP, prostatic acid phosphatase.

a higher risk of developing pre-malignancies/malignancies. Various types of pre-malignancies were reported, such as hyperplasia of breast and prostate, polyp proliferative lesions, suspected liver/lung malignant tumor lesions, Helicobacter pylori positivity, cirrhosis, HBV positivity and chronic inflammatory diseases as well as benign tumor, obesity and fatty liver. Follow-up data (3-6 years) of individuals with elevated STK1p values $(\mathrm{n}=170$; STK1p $>2.0 \mathrm{pM})$ showed a 3- to 5-fold higher risk of developing malignancies, compared to individuals with normal STK1p values $(n=6,354$; risk cut-off value $<2.0 \mathrm{pM})$ (26). However, additional long-time (10-15 years) follow-up studies, some already in progress, are required to eventually prove the applicability of STK1p, identifying individuals at risk of developing cancer, in health screening.

The percentage of individuals with elevated STK1p values in a group of city-dwelling individuals was $0.8 \%(n=26,484)$. Approximately $85 \%$ of those individuals had diseases associated with processes with a potential to lead to malignancies or had already established malignancies at higher frequencies compared to individuals with healthy STK1p values, indicating statistical significance $(\mathrm{P}<0.01)$. The frequency of individuals with elevated STK1p values markedly varied in different cities (0.5-1.1\%), possibly reflecting differences in living and working conditions. The frequency of individuals with elevated STK1p values was also higher than the cancer incident rate in China (0.2-0.5\% incident rates) (72), possibly because patients with elevated STK1p values showed pre-malignancies, although not all pre-malignancies become malignancies. Furthermore, the frequency of individuals with malignancies in these health screenings was low $(\sim 0.01)$ since in China most individuals with symptoms of cancer directly contacted oncology clinics instead of health screening centres.

In contrast to those living in cities, individuals living and working in a land-based oil field $(n=8,869)$ showed a significantly higher frequency of elevated STK1p values (5.8\%), demonstrated by results of the tests conducted at the Health Centre of the General Hospital of the Jilin Oil-field, Jilin, China (24). Although the same high frequency of pre-malignancy/malignancy was observed as for the city-dwelling individuals $(\sim 85 \%)$ in the group of patients with higher STK1p values, they had a significantly higher frequency of refractory anaemia, fatty liver and obesity. The refractory anaemia frequency was $\sim 40 \%$, compared to $\sim 1 \%$ among the city-dwelling individuals. Anaemia is one of the pre-cancerous myelodysplasia diseases with the potential to develop into leukaemia $(72,73)$. The high frequency of refractory anaemia among the oil-field workers is likely to have been induced by benzene toxicity $(74,75)$. However, there were hardly any workers with breast and prostate hyperplasia, compared to the city-dwelling group $(\sim 50 \%)$. Furthermore, individuals exposed directly or experiencing a long exposure to oil-derived pollutants, including drilling, transportations and geological prospecting, had a higher frequency of STK1p values (7.8\%), compared to individuals working at the service/administration department of the oil-field company (3.9\%), indicating a statistically significant difference. Consequently, the level of STK1p in the worker group reflects a higher risk of developing cancer in the haematopoietic 
Table II. STK1p in relation to clinical parameters.

\begin{tabular}{lcccccccc}
\hline Type of tumor & Clinical stage & Grade & $\begin{array}{c}\text { Monitoring } \\
\text { treatment }\end{array}$ & $\begin{array}{c}\text { Clinical outcome } \\
\text { of treatment }\end{array}$ & Relapse & Survival & Benign & Healthy \\
\hline Breast & Yes & Yes & Yes & Yes & Yes & ND & $>$ & $>$ \\
Cervical & Yes & Yes & ND & ND & ND & Yes & ND & $>$ \\
Esophagus & Yes & No & Yes & Yes & ND & ND & ND & $>$ \\
Gastric and cardiac & Yes & No & Yes & Yes & ND & ND & $>$ & $>$ \\
Colorectal & ND & ND & Yes & ND & ND & ND & ND & $>$ \\
Renal cell carcinoma & No & No & ND & ND & ND & ND & No diff. & No diff. \\
Bladder & Yes & No & Yes & ND & ND & ND & ND & $>$ \\
Prostate & Yes & ND & ND & ND & ND & ND & $>$ & $>$ \\
Head and neck & ND & ND & Yes & Yes & ND & ND & ND & $>$ \\
Lung & Yes & Yes & Yes & Yes & ND & ND & ND & $>$ \\
Leukaemia & ND & ND & Yes & Yes & ND & ND & ND & $>$ \\
Lymphoma & ND & ND & Yes & Yes & ND & Yes & ND & $>$ \\
Hepatoma & ND & ND & ND & ND & ND & ND & ND & $>$ \\
Brain & ND & ND & ND & ND & ND & ND & ND & $>$ \\
\hline
\end{tabular}

ND, not determined; >, STK1p values are significantly higher in malignant patients compared to benign and healthy individuals; No diff., No difference in STK1p values between malignant patients and benign and healthy individuals.

system (anaemia) $(74,75)$, as a result of exposure to pollutants coupled with oil production.

STK1p in clinical oncology. The significance of STKa for the prognosis, monitoring of tumor treatments, relapse and survival has been discussed in previous reviews (1,3-5). In the present review, the applicability of STK1p in clinical oncology is discussed. The results are based on case-control clinical trials or routine clinical settings. Some of the results were obtained from clinical pilot studies. In a routine clinical setting patients were analyzed upon arrival to the laboratory without any planned study. The analysis, however, was carried out in accordance with the approved clinical laboratory practices. In a clinical trial the medical parameters of the particular type and schedule of treatments must be strictly followed. In routine clinical settings, however, it is not always possible to follow recommendations from clinical trials, which may result in unreliable prognosis. Therefore, whether it is possible to draw conclusions concerning the applicability of STK1p from routine clinical settings should be investigated. However, close correlations between STK1p values and clinical parameters including the outcome of treatments and survival were found, although results from routine clinical settings require further confirmation. In the studies discussed in the present review, healthy individuals of relevant gender and ages were used as the controls. STK1p in association with various types of malignancies is shown in Table II.

\section{Gynaecology}

\section{Breast cancer.}

STKIp as a marker for monitoring treatment. As part of a clinical trial, STK1p was examined in patients with non-lymph node metastatic breast cancer pre- $(n=17)$ and post-operation $(n=38)$, as well as in patients with lymphnode metastasis $(n=10)$, compared to patients with benign tumors $(n=21)$ as well as healthy individuals $(n=11)(15)$. Significantly higher STK1p values were evident prior to surgery compared to 3 months after surgery. In patients with lymph-node metastasis, STK1p was significantly higher compared to patients with no metastasis. The benign patients showed a significantly lower STK1p compared to pre-operative breast cancer patients. The STK1p values of the healthy individuals were significantly lower compared to both the breast cancer patients and the benign patients. A series of similar studies have demonstrated that STK1p has the potential to be a reliable biomarker for monitoring treatments (28-32).

In a recent study on breast carcinoma patients conducted by Carlsson et al (31), the STK1p levels were determined subsequent to surgery $(n=24)$ and chemotherapy $(n=39)$ by a non-radioactive immunological ELISA TK1 assay using the same anti-TK1 immunoglobulin Yolk (IgY) antibody as provided in the STK1p dot blot assay by SSTK Inc. Although the ELISA assay is less sensitive compared to the ECL dot blot assay, STK1p values were significantly reduced 4 weeks after the surgery. The STK1p significantly increased after tumor recurrence and decreased again following additional chemotherapy in the recurrent patients.

In a follow-up study on neo-adjuvant-treated breast cancer patients $(n=40)$, the STK1p levels were positively correlated with variables such as histological grade, clinical stage, tumor size and C-erbB-2, although negatively correlated with oestrogen receptor (ER). Subsequent to neo-adjuvant therapy, the STK1p values of stage II patients showing complete (CR) and partial response (PR) decreased by 64 and 78\%, respectively, whereas 
only by $17 \%$ in patients with no response (NR). $\mathrm{P}=0.013$ was considered to indicate a statistically significant difference. Similar results were found in the stage III patients, with the exception that fewer patients showed decreasing STK1p values $(50 \%)$. Thus, STK1p may be used to evaluate the effect of neo-adjuvant chemotherapy in breast carcinoma patients (32).

STK1p and recurrence. The STK1p value was used as a predictive biomarker for recurrence in a case control trial on low-risk breast cancer patients for a follow-up period of 11 years $(n=120)(20)$. The STK1p values 3 months after surgery were significantly higher in patients with a longer recurrence. A multivariate analysis showed a 6 -fold higher risk of developing recurrence in the STK1p-elevated group.

STK1p in combination with SCT-scanning. In a breast carcinoma study conducted by Lu et al (33), the STK1p values were determined and compared to the size of the tumor measured by means of enhanced CT-scanning (SCT). The STK1p level of the breast carcinoma patients was significantly higher compared to that of patients with breast benign/pre-malignant tumors. The correct diagnostic rate of breast carcinoma patients determined by SCT was $92.1 \%$, and $71.1 \%$ by STK1p. When combined with the STK1p values the diagnostic rate of SCT increased to $97.3 \%$. The correct diagnostic rate of breast benign and pre-malignancy patients determined by SCT was $82.4 \%$, whereas $94.1 \%$ by STK1p. STK1p values were positively correlated with the tumor size, degree of enhancement and axillary lymph-node invasions $(\mathrm{P}<0.05)$. The authors concluded that the SCT in combination with the STK1p assay increased the accuracy of the prognosis of neoplasms, while providing a more precise parameter for clinical treatment.

Serological TK activity. Recently, Nisman et al (74) have also reported that STKa is an important risk factor, indicating a high proliferation potential of tumors at the time of excision. Multivariate COX proportional hazard analyses demonstrated that STKa adjusted for stage, grade, necrosis, and ER and PR negativity was retained as an independent predictor of disease recurrence (hazard ratio, 3.9; 95\% CI, 1.3-11.6; $\mathrm{P}=0.013$ ). STKa was reported for the first time to correlate with BRCA $1 / 2$ mutations.

\section{Cervical cancer.}

Cervical cancer is the third most common malignancy in women and a major cause of morbidity and mortality, particularly in developing countries. The abnormalities in cervical cells occur in the squamous epithelium and are considered to be pre-cancers of invasive squamous carcinomas. As yet, no specific prognostic tumor markers or markers with the potential to be used for follow-up survival are known for cervical carcinoma.

The prognostic value of STK1p and the use of STK1p for monitoring the effect of treatment were examined in cervical carcinoma patients $(n=85)$ and in patients with cervical intraepithelial neoplasia (CIN, $n=30$ ), and compared to healthy women $(n=41)$. The STK1p values in the cervical carcinoma patients were significantly higher compared to CIN patients and healthy women $(\mathrm{P}<0.05)$. The STK1p levels increased significantly from stage I-II to III-IV $(\mathrm{P}<0.05)$. The STK1p values were reduced after surgery to values close to those in healthy women $(\mathrm{P}<0.05)(34)$.
In another study on patients with locally advanced cervical carcinoma $(\mathrm{n}=45)$, STK1p was compared to SCC-Ag, prior and subsequent to treatment with synchronous chemo/radiotherapy. SCC-Ag is a serum marker used for monitoring, but not for follow-up survival, in patients with cervical cancer. The positive rates of STK1p (>2.0 pM) and SCC-Ag were 51.1 and $68.9 \%$, respectively, and were closely associated with clinical stages, lymph-node metastasis and tumor size $(\mathrm{P}<0.05)$. The positive rates of STK1p and SCC-Ag significantly decreased to 14.6 and $18.6 \%$, respectively, after one cycle of treatment. Thus, STK1p may be used for prognosis in patients with locally advanced cervical carcinoma and for evaluating the effects of the treatment (35).

\section{Digestive system}

Esophageal cancer. Esophageal cancer is one of the two most frequent types of malignancies in China. STK1p was evaluated as a prognostic factor in esophageal carcinoma patients in two studies, $(36, n=101)$ and $(37, n=363)$. In the first study, the STK1p values were highly correlated with clinical stages $(0+\mathrm{I}$ vs. stage III+IV; P=0.005). Although STK1p was not correlated with tumor size, a significant increase in STK1p values was observed from $\mathrm{T} 1$ to $\mathrm{T} 4(\mathrm{P}=0.021)$. The STK1p values of patients with lymph-node metastasis were higher compared to patients without lymph-node metastasis $(\mathrm{P}=0.065)$. In the present study, the STK1p value was not correlated with pathological grades (36).

In the second study, STK1p values decreased by 45 and/or $71 \%$ subsequent to surgery and chemotheraphy, respectively $(\mathrm{P}=0.001)$. Subsequent to treatment, STK1p values also varied depending on the presence or absence of metastasis, i.e., STK1p decreased to $0.9 \mathrm{pM}$ in non-metastatic patients, while remaining high in metastatic patients (3.9 pM) (37).

Gastric and cardiac cancer. STK1p and STKa levels were determined in pre- and post-surgery gastric cancer patients $(n=43)(16)$. One month subsequent to surgery, the STK1p level decreased by $\sim 50 \%$. At 3 months the STK1p value was almost back to normal. By contrast, there were no significant changes in the STKa values. Recent studies on gastric cancer patients, partly based on routine clinical settings, confirmed these results $(36,38-40)$. For example, in one of these studies $(n=102)$, the STK1p values decreased to normal in patients without metastasis within 1 month after surgery, while remaining high in patients with metastasis (37). The number of STK1p-positive patients $(>2.0 \mathrm{pM})$ prior to surgery was significantly higher compared to other digestive markers. In a study by Chen et al (36) (n=69), STK1p was found to correlate with clinical stages, partly with tumor size and treatment (chemotherapy, radiotherapy or biological therapy) outcome (STK1p was lower in $\mathrm{CR} / \mathrm{PR}$ compared to SD/PD). Thus, STK1p may be used as a marker for monitoring the response to treatment 1 month after surgery in patients with gastric carcinoma.

In a comparison study, STKa was markedly elevated in gastric adenocarcinoma compared to healthy individuals $(\mathrm{n}=72)(75)$.

Colorectal. Colorectal cancer is a common malignant tumor of the digestive system. Mild symptoms presenting at an early stage of the tumor often pass unnoticed by both patients and physicians, whereas when clinical symptoms become more distinct, patients are likely to be at advanced 
stages. Thus, several studies have been conducted on STK1p in comparison with other biomarkers (41-45).

In a study on 140 patients STK1p was compared to CEA, a commonly used serum marker in colorectal patients (44). The number of positive STK1p (>2.0 pM) and CEA patients was 63.8 and $59.3 \%$, respectively. The percentage of TK1 in immunohistochemistry showed a labeling index (LI) of $78 \%$. The level of STK1p decreased gradually at 1 and 3 months after surgery and reached normal levels at 6 months (42).

To evaluate the benefits of laparoscopic surgery over open surgery, STK1p, EGF, HGF, CRP, and IL-6 markers were used to assess the clinical outcome $(n=66)$. These markers showed a more rapid decrease 1 month after laparoscopic surgery, compared to open surgery $(\mathrm{P}<0.05)(43)$.

The number of polyps, distribution, size and shape, as well as the pathological types of colorectal cancer is directly associated with the development of malignancies. In a study on polyps $(n=122)(45)$, significantly higher expression levels of STK1p were found in polyp groups of adenomatous, sessile colorectal and $\geq 2 \mathrm{~cm}$ polyp group, compared to the polyp group of non-adenomatous, pedunculated colorectal, 1 polyp group and healthy individuals. Thus, STK1p may be used to assess the proliferation rate of different colonic polyp patient groups, indicating an early risk of developing cancer in the future.

\section{Urinary system}

Prostate carcinoma. Prostate carcinoma is one of the most common cancer types of the urinary system, and onset is increasingly observed in younger age groups. The first comparison study on STKa and PSA was performed in 1996 $(n=92)$ (76). STKa was found to be a more reliable marker compared to PSA. Recently, STK1p was also shown to be a reliable marker in prostate carcinoma patients (46). STK1p had the potential to distinguish between patients with prostate carcinoma $(n=70,3.66 \pm 1.91 \mathrm{pM}$, sensitivity 0.72 at a cut-off value of $2.0 \mathrm{pM})$ and patients with prostate hyperplasia $(n=40$, $1.28 \pm 0.35 \mathrm{pM})(\mathrm{P}<0.05)$, indicating a statistically significant difference. The STK1p value in healthy individuals $(n=40)$ was $0.79 \pm 0.27 \mathrm{pM}$. The positive rate of STK1p $(>2.0 \mathrm{pM})$ was associated with clinical stages $\left[\mathrm{T}_{1} 62.9 \%(22 / 35), \mathrm{T}_{2} 73.7 \%\right.$ (14/19), $\mathrm{T}_{3} 80.0 \%(8 / 10)$, and $\mathrm{T}_{4} 100.0 \%$ (6/6)]. Thus, STK1p is a potential biomarker in patients with prostate carcinoma capable of distinguishing between prostate hyperplasia and carcinoma.

Renal cell carcinoma (RCC). The STK1p level of RCC patients was low with no statistically significant difference in healthy individuals, while the STKa level was significantly higher $(50, n=26)$. STKa was correlated with clinical stage, grade and tumor size. However, in a recent study, the STK1p value was significantly higher in patients with RCC (positive rate $60.0 \%$ ) compared to healthy individuals (47).

The use of STKa as a prognostic biomarker in RCC patients $(\mathrm{n}=116)$ was confirmed by Nisman et al $(77)$. STKa was significantly higher compared to healthy individuals, but not to patients with benign kidney tumors $(n=27)$. STKa was correlated with $\mathrm{T}$ stage, although not with grade. The presence of extensive tumor necrosis ( $>50 \%)$ was statistically correlated with a high TuM2-PK and a low STKa level. TuM2-PK is a marker for the overall cell metabolism. High levels of STKa and TuM2-PK were correlated with reduced 5-year recurrence-free survival. Multivariate Cox analysis showed that STKa and TuM2-PK were independent predictors of recurrence.

Bladder cancer. STK1p was significantly higher in patients with bladder carcinoma, compared to healthy individuals (19). Following surgery, STK1p decreased rapidly within 1 week and returned to normal values 1 month after the surgery. The patients were considered to be tumor-free, when the STK1p values were normalized.

Head and neck cancer. Head and neck cancer includes a number of different types of malignancies, for example, tongue, pharynx, parotid gland, larynx, nasopharynx, tonsil, facial and gum. Since head and neck cancer is complex, useful biomarkers for prognosis and monitoring of treatment remain to be identified. However, STK1p was found to be a reliable marker for monitoring the therapy of head and neck cancer patients $(n=138)(36)$, as reported for the first time in 2010. The STK1p level decreased significantly after surgery or chemotherapy, individually or in combination. The decrease in STK1p values was notably more obvious following the combined treatment as compared to individual treatment. STK1p was also found to be correlated with the clinical reaction to treatments (STK1p of PD patients $>$ STK1p of CR/PR patients) Thus, STK1p is likely to be a potential biomarker in distinguishing between the outcome of surgery and/or chemotherapy in patients with head and neck cancer.

Lung cancer. STK1p was found to be a reliable marker for the prognosis and monitoring of the treatment of non-small cell lung carcinoma (NSCLC) patients $(n=250)(17)$. STK1p levels decreased significantly after surgery in non-metastatic patients, but not in patients with metastasis, and increased significantly with T-values and with clinical stage and invasiveness. However, no difference was detected in STK1p values between SCC and adenocarcinoma (AC) types of tumor. Chen et al (36) and Li et al (37) confirmed these results. STK1p was also found to be correlated with the outcome of the therapy (surgery and/or chemotherapy) (STK1p $<\mathrm{CR}<\mathrm{PR}<\mathrm{SD}<\mathrm{PD}$ ) and with grades, lymph nodal status and tumor size (36).

Moreover, STK1p was compared to CYFRA21-1 and NSE in patients with lung cancer (51). CYFRA21-1 and NSE are serum markers commonly applied in lung cancer patients. The three sera markers were significantly higher compared to healthy individuals. The positive rates of STK1p, CYFRA21-1 and NSE were $74.4,51.2$ and $46.5 \%$, respectively. Thus, STK1p provides useful and probably more reliable information on tumor cell proliferation in lung cancer, compared to CYFRA21-1 and NSE.

Biomarkers alone or in combination have the potential to be used for the clinical setting of treatment of lung cancer (52-54). For example, STK1p, CEA, CA-125 or CA-199 were used in a 6-18 month follow-up study $(n=24)$. The STK1p value decreased significantly after treatment. The positive rates of these markers alone or in combination were found to be correlated with the treatment outcome as follows: STK1p (91.7\%), CA-125 (86.7\%), CEA (63.6\%), CA-199 (61.6\%), STK1p + CA-125 (100\%), STK1p + CEA (95.8\%) and STK1p + CA-199 (95.8\%) (52). 
Haematological diseases. The main feature of haematological diseases is cell proliferation. STKa has been a useful tool in detecting these types of malignancies $(1,3,4)$. Furthermore, STK1p has also been reported to have prognostic significance in patients with chronic lymphocytic leukaemia (CLL) $(52,53)$. STK1p was correlated with other prognostic factors frequently used in the detection of this type of disease. The STK1p level was significantly elevated in patients with Binet $\mathrm{C}$, higher levels of serum lactate dehydrogenase (LDH) and b2-microglobulin (b2-MG), un-mutated immunoglobulin heavy chain variable (IGHV) status, higher expression levels of ZAP-70 and CD38, compared to patients with Binet A, lower levels of serum LDH and b2-MG, mutated IGHV status, lower expression levels of ZAP-70 and CD38. A strong correlation was observed between the STK1p level and IGHV mutations or ZAP-70. According to results of the ROC curve analysis of the STK1p and IGHV mutational statuses, the area under the curve was $0.757(\mathrm{P}=0.001)$ at an optimal cut-off value of a STK1p level of $1.75 \mathrm{pM}$, with a sensitivity of 0.64 and a specificity of 0.88 . High STK1p levels predict a poorer overall survival. STK1p was also found to correlate with un-mutated immunoglobulin variable region genes, expression of CD38 and ZAP-70, as well as the subsequent risk of developing a large B-cell lymphoma (Richter syndrome). Konoplev et al (78) confirmed these results when using TK activity in serum. Their findings showed that in a univariate COX analysis only Richter syndrome and STKa were significant prognostic factors for overall survival. Thus, STK1p is a predictive biomarker in CLL patients and may be used to assess prognosis in these types of patients. In addition, these results confirm that the dot blot chemiluminescent assay $(52,53)$ and TK-REA yielded corresponding results (78).

Zeng et al (54) showed for the first time that STK1p may be used for monitoring tumor therapy in leukaemia. The levels of STK1p were markedly different in patients with NR $(n=22)$, PR $(n=20)$ and CR $(n=8)$. Furthermore, no statistically significant differences were found in STK1p values in CR patients and healthy individuals, indicating the successful removal of the tumors by the treatment. These results confirm that STK1p has the potential to be used for assessing chemotherapy and providing guidance for the adjustment of therapy in patients with leukaemia.

For the first time, Pan et al (55) showed that STK1p is useful in predicting the outcome after treatment with CHOP chemotherapy in patients with non-Hodgkin's lymphoma with follicular small cleaved cell $(n=13)$, follicular large cell $(n=6)$ and diffused large cell $(n=18)$. The STK1p level prior to and at 1 day and 4 weeks after chemotherapy was found to vary significantly, while being correlated with the clinical response $(\mathrm{CR}<\mathrm{PR}<\mathrm{NR})$ and 5-year survival rate. These findings indicate that STK1p may be of marked importance in the evaluation of the treatment outcome and prediction of recurrence in patients with non-Hodgkin's lymphoma. In a similar study on follicular lymphoma (79) $(n=170)$ STKa of pre-treated patients was found to be a potentially independent predictive factor of overall and progression-free survival (hazard ratio $2.91 ; \mathrm{P}=0.019$ ), as well as in ages above 60 years (hazard ratio $5.21 ; \mathrm{P}<0.001)$.

Other types of malignancies. A limited number of studies has been conducted on rectal carcinomas and carcinomas of the hepatoma and the brain, showing significantly higher STK1p values compared to healthy individuals (18). The STK1p values of these patients regarding prognosis and follow-up survival are currently under investigation.

\section{The benefit of chicken IgY antibody in serum assay}

The benefit of chicken antibodies (IgY) is that they do not activate the human complement system, a well-known source of interference in ELISA sandwich immunoassays (31). Capture antibodies bound to a solid surface are potent activators of the complement factors of an antibody, with the activated complement factors reacting with the assay antibodies, thus partly blocking the antigen-binding sites. Another advantage is that rheumatoid factors (RF) do not bind to IgY. $\mathrm{RF}$ is a major source of interference in many immunoassays, reacting with the Fc-portion of mammalian $\mathrm{IgG}$. The disease usually associated with RF is rheumatoid arthritis, although $\mathrm{RF}$ is also present in blood samples of patients with several additional diseases, as well as of healthy individuals. Most immunoassays using mammalian poly- or monoclonal antibodies are subjected to RF binding, thus potentially providing false-positive results. As RF does not bind to IgY, chicken antibodies are useful in immunoassays, whereby RF might interfere. Another interfering factor is human anti-mouse IgG antibody (HAMA). An increasing number of patients with various diseases are treated in vivo with monoclonal mouse antibodies, often inducing an antibody response in the patient, resulting in HAMA production. Chicken antibodies do not react with HAMA, thus having the potential to be used to eliminate such interference. Consequently, chicken antibodies theoretically have the advantages over immunoassays containing mammalian antibodies, especially for tumor marker assays, when mouse monoclonal antibodies were used for tumor treatment, increasing the prevalence of HAMA.

Thus, serological TK1 concentration alone or in combination with routine health exam systems or other tumor biomarkers has the potential to identify individuals with pre-cancerous lesions or early malignancies (56-59). STK1p is also useful for the prognosis and monitoring of treatment, relapse and survival in cancer patients. The highly sensitive chemiluminescent STK1p dot blot assay is a cost-effective test, making it attractive to use in health screening, particularly in developing countries.

\section{TK1 immunohistochemistry in clinical oncology}

Although TK1 is known to be closely correlated with cell proliferation, the lack of useful anti-TK1 antibodies did not promote TK1 immunohistochemistry. Using the monoclonal TK1 antibody produced by our Sino-Swed TK1 research group, Gasparri et al (14) compared the expression of TK1 with Ki-67, the most commonly used proliferation marker in oncology at present. The expression was determined by using high-content ArrayScan ${ }^{\mathrm{TM}}$ fluorescence microscopy. The expression of TK1 started to increase 6-7 h subsequent to serum re-addition to starved human normal dermal fibroblast cells, i.e., when the cells were still in the G1 stage of the cell cycle. This increase occurred earlier than the onset of the Ki-67 expression and hours before the initiation of the DNA-synthesis (BrdU incorporation). Thus, a population of activated G1 cells with higher TK1 compared to Ki-67 expres- 
Table III. TK1 expression of tumor tissues in relation to clinical parameters.

\begin{tabular}{lcccccccc}
\hline Type of tumor & Stage & Grade & Relapse & Survival & Benign & Health & PCNA & Ki-67 \\
\hline Breast & Yes & Yes & ND & ND & $>$ & $>$ & $>$ & $>$, no diff. \\
Cervical & Yes & Yes & ND & Yes & ND & $>$ & ND & $>$ \\
Ovary & Yes & Yes & Yes & Yes & ND & $>$ & ND & No diff. \\
Lung & Yes & Yes & ND & Yes & $>$ & $>$ & ND & ND \\
Colorectal & Yes & No & ND & ND & ND & $>$ & $>$ & ND \\
Renal cell carcinoma & Yes & Yes & ND & ND & ND & $<,>$ & ND & $>$, no diff. \\
Prostate & Yes & ND & ND & ND & $>$ & $>$ & ND & ND \\
\hline
\end{tabular}

ND, not determined; $>/<$, TK1 expression is higher/lower in malignant patients compared to benign and healthy individuals; no diff., no difference in TK1 expression between malignant patients and benign and healthy individuals. PCNA, proliferating cell nuclear antigen.

sion were identified, showing that TK1 should detect a higher proportion of proliferating cells compared to Ki-67. This was found in patients with cervical and breast carcinomas, although not in patients with ovarian carcinoma or RCC. The results of the TK1 expression in tumor tissues during the last decade are shown in Table III.

Breast carcinoma. The TK1 expression was examined in three independent clinical studies and compared to the expression of proliferating cell nuclear antigen (PCNA) (22) and Ki-67 $(62,63)$. TK1 was found mainly in the cytoplasm, however, only to some extent in the nucleus. TK1 expression (LI) was significantly higher compared to that of PCNA (60), as well as higher in one of the two Ki-67 studies compared to Ki-67 (62). The TK1 expression was significantly correlated with stage and grade $(60,62)$, particularly in stage II and grade II (62). To investigate the possibility of using the TK1 expression to confirm the diagnosis in pre-malignant breast cancer patients, TK1 expression was determined in patients with usual ductal hyperplasia (UDH), atypical ductal hyperplasia (ADH), ductal carcinoma in situ (DCIS) and invasive ductal carcinoma (IDC) (64). TK1 expression was found to significantly increase in the breast ductal carcinomas as follows: $\mathrm{UDH}<\mathrm{ADH}<\mathrm{DCIS}<\mathrm{IDC}$. TK1 expression was correlated with the histological grade in DCIS and IDC patients, as well as with the pathological stage in IDC patients. The degree of TK1-positive rates (LI) in the tumors of patients with ADH, DCIS and IDC was $80-90 \%$, while the corresponding value in UDH patients was $<5 \%$. Expression of TK1 and Ki-67 showed extremely high correlations in the four tumor groups investigated. Since the TK1 expression varied significantly in the breast ductal subgroups, TK1 was confirmed to be a reliable proliferation marker for the determination of breast tumor proliferation, particularly in pre-cancerous lesions (ADH). This finding is likely to enable timely treatment in early stages of tumor development using minimal access surgery, thus avoiding extensive radical breast surgery and improving survival rates and the quality of life.

Cervical lesions. The expression of TK1 and Ki-67 was investigated in pre-malignant and malignant lesions of cervix (64). TK1 was found both in the cytoplasm and the nucleus. The LI of TK1 was significantly higher compared to Ki-67 in CIN $(n=216)$ and cervical carcinoma $(n=84)$ patients. Both the LI and intensity of the TK1 expression increased with the CIN grades and pathological stages. The LI of Ki-67 was also correlated with CIN grades and pathological stages, although there was no change in the intensity of the expression. A high TK1 expression in the nucleus was correlated with a poor 5-year survival in invasive cervical carcinoma patients, compared to patients with a lower nuclear TK1 expression. Notably, in invasive cervical carcinoma patients in the pathological stage III/FIGO stages IIA-IV with poor prognosis, there was a group of patients $(\sim 50 \%)$ with a low TK1 expression with good survival ( $\sim 80 \%$ survival rate). The Ki-67 expression did not reflect this finding. Multivariate COX analysis showed that an increased TK1 expression in the nucleus was an independent prognostic factor in invasive carcinoma patients, although this was not the case for Ki-67. Thus, the TK1 expression in the nucleus of cervical lesions is a reliable prognostic factor for the identification of the risk of developing malignancy in CIN patients at an early stage and predicting the survival in invasive cervical carcinoma.

Ovary carcinoma. Expression of TK1 and Ki-67 was studied in ovarian serious adenocarcinoma $(n=55)(65)$. The LI of TK1 and Ki-67 were 72.2 and $80.0 \%$, respectively. TK1 was detected in the cytoplasm. TK1 and Ki-67 were significantly correlated with pathological stage and grade, tumor size, recurrence and a 10-year survival. Multivariate analysis showed that TK1, but not Ki-67, was an independent prognostic marker of stage, grade and age. Patients with advanced tumor and low TK1 expression had a better survival compared to patients with a high TK1 expression. Thus, a high TK1 expression indicated a high risk of recurrence and poor survival, suggesting intensive therapy and frequent follow-up.

Lung carcinoma. The expression of TK1 and Ki-67 was compared in NSCLC of AC and SCC types (63). The LI of TK1 was significantly higher $(68 \%)$ in AC patients compared to Ki-67 (36\%), although no such difference was detected in SCC patients. Thus, TK1 is a more reliable proliferation marker than Ki-67 in AC patients, but not in SCC patients.

In a second study on pT1 lung adenocarcinoma (66), the 5 -year survival was significantly less frequent in patients with a high TK1 expression (LI $>25 \%$ ) compared to patients with a low TK1 expression (LI <25\%). TK1 expression and tumor stage were found to be independent prognostic factors. Additionally, patients with a stromal invasion grade III are 
normally expected to have poor 5-year survival. TK1 expression had the potential to identify pT1 patients of stromal invasion grade III with a low TK1 expression and good survival, rendering it possible to further improve the treatment of these patients, possibly prolonging their survival or even curing them. This is the first study to show that the TK1 expression in combination with stromal invasion is a more reliable prognostic factor compared to stromal invasion classification itself, in patients with pT1 lung adenocarcinoma.

Colorectal carcinoma. Expression of TK1 and PCNA was studied in 74 patients with colorectal malignancy (62). The LI of TK1 was higher (65\%) compared to PCNA (52\%), although without a statistically significant difference $(\mathrm{P}=0.171)$. The markers showed a significant difference between colorectal carcinoma and colorectal adenoma and were markedly correlated with stage, although TK1 was correlated with the grades only.

In a recent study, the TK1 expression was investigated in relation to different types of colon polyps $(n=122)(45)$. TK1 expression was significantly higher in polyp groups of adenomatous, sessile colorectal, as well as $\geq 2 \mathrm{~cm}$ polyp group, compared to the polyp group of non-adenomatous, pedunculated colorectal, 1 polyp group and healthy individuals. Therefore, TK1 expression has the potential to assess the proliferation rate of various colonic polyp patient groups, as found for STK1p, and provide an early warning of risk of developing cancer in the future.

$R C C$. Luo et al (67) examined the expression of TK1 and Ki-67 in RCC $(n=26)$. Immunohistochemistry and western blot analysis showed that TK1 expression levels depend on the source of RCC. Comparison of the expression of TK1 in healthy kidney tissue to RCC tissue showed that the healthy tissue had a higher TK1 expression, due to the high expression of TK1 in tubular cells. These results confirmed the findings of recent studies showing that tubular cells of healthy kidney tissues are in an active G1 stage of the cell cycle. The low expression of TK1 in renal carcinoma cells indicated the low proliferation rate of this type of malignancy. Although no difference was found in the expression of TK1 and Ki-67 in RCC patients in healthy kidney tissues the Ki-67 expression was absent. In their study, Gakis et al (68) detected a higher TK1 expression in RCC tumor tissues compared to oncocytomas and healthy kidney tissues. However, the TK1 expression level in RCC varied depending on the RCC type, with a higher TK1 expression in the ccRCC and papRCC types, while the TK1 expression in the chRCC type was similar to that of healthy kidney tissues. TK1 was mainly detected in the cytoplasm. TK1 expression was also correlated with stage and grade. There was a trend, although not statistically significant, for the TK1 expression to be higher in a smaller tumor $(<7 \mathrm{~cm})$ compared to a larger one $(>7 \mathrm{~cm})$, likely due to a lower proliferation rate in larger tumor of reduced nutrition supply. Since the individual biological aggressiveness of RCC is almost unpredictable, the authors of the present study concluded that TK1 expression in RCC tumor tissues may provide additional information to characterize the patient-risk profiles and determine individual therapy.

Prostate carcinoma. Ye et al (69) investigated the expression of TK1 and Ki-67 in patients with prostate carcinoma $(n=42)$, prostate intraepithelial neoplasia (PIN) $(\mathrm{n}=35)$, benign prostate hyperplasia $(\mathrm{BPH})(\mathrm{n}=25)$ as well as in individuals with healthy prostate tissues $(\mathrm{n}=10)$. Almost no expression of TK1 was detected in BPH and healthy prostate tissues, while $17.0 \%$ of patients with PIN and $57.1 \%$ of patients with prostate carcinoma demonstrated TK1 expression. The LI of Ki-67 in the prostate carcinoma patients was $47.6 \%$. Thus, TK1 expression has the potential to be used as a cell proliferation index in prostate carcinoma patients and to provide valuable information on treatment and prognosis. In a recent study (70), the anti-TK1 mAb developed by SSTK Inc., (clone 5) was found to induce specific staining in prostate carcinoma tissue. TK1 $\mathrm{mAb}$ is therefore useful for an accurate histopathological differentiation between prostate carcinoma and healthy prostate tissue. Moreover, TK1 expression provided additional information on the recurrence and metastatic behaviour, having the potential to identify highrisk patients and to perform tailor-made treatment regimens. In a multivariable analysis, an independent association between TK1, T-stage and the development of metastatic disease was detected (hazard ratio, 1.01; $\mathrm{P}=0.0007$ ) (70).

\section{Conclusion}

Since 1980, TK1 has been used as a clinical marker for tumor proliferation based on TK activity in serum, mostly in patients with lymphoma and leukaemia, and to some extent in patients with breast carcinoma. TK1 has almost no immunohistochemistry, due to a lack of useful anti-TK1 antibodies. The recent development of new-generation chicken anti-TK1 (IgY) and mouse monoclonal anti-TK1 antibodies improved extensively the clinical use of TK1 in almost every type of solid human tumors, for both serum and immunohistochemistry analyses. Based on the clinical studies using the new generation of TK1 antibodies that were conducted thus far, TK1 is likely to become the recommended marker for the prognosis as well as the monitoring of treatment and follow-up in cancer patients in the next 5-10 years. TK1 is also expected to be used in health screening to predict the risk of future cancer development in individuals with pre-cancerous and/or cancer-risk diseases. The sensitive chemiluminescent TK1 assay is a useful tool, due to its high sensitivity and specificity [ROC-value 0.96 , likelihood (+) value 199.7], as well as its being an efficient and cost-effective assay.

\section{References}

1. Gronowitz JS, Hagberg H, Kallander CF and Simonsson B: The use of serum deoxythymidine kinase as a prognostic marker, and in the monitoring of patients with non-Hodgkin's lymphoma. $\mathrm{Br}$ J Cancer 47: 487-495, 1983.

2. Wu CJ, Yang RJ, Zhou J, Bao S, Zou L, Mao YR and He Q: Production and characterisation of a novel chicken IgY antibody raised against $\mathrm{C}$-terminal peptide from human thymidine kinase 1. J Immunol Methods 277: 157-169, 2003.

3. O'Neill KL, Buckwalter MR and Murray BK: Thymidine kinase: diagnostic and prognostic potential. Expert Rev Mol Diagn 1: 428-433, 2001.

4. Topolcan $\mathrm{O}$ and Holubec L Jr: The role of thymidine kinase in cancer diseases. Expert Opin Med Diagn 2: 129-141, 2008.

5. von Euler H and Eriksson S: Comparative aspects of the proliferation marker thymidine kinase 1 in human and canine tumour diseases. Vet Comp Oncol 9: 1-15, 2011.

6. He E, Xu XH, Guan H, Chen Y, Chen ZH, Pan ZL, Tang LL, $\mathrm{Hu}$ GZ, Li Y, Zhang M, et al: Thymidine kinase 1 is a potential marker for prognosis and monitoring the response to treatment of patients with breast, lung, and esophageal cancer and nonHodgkin's lymphoma. Nucleosides Nucleotides Nucleic Acids 29: 352-358, 2010. 
7. Aufderklamm S, Todenhöfer T, Gakis G, Kruck S, Hennenlotter J, Stenzl A and Schwentner C: Thymidine kinase and cancer monitoring. Cancer Lett 316: 6-10, 2012.

8. James T Wu: Circulating Tumor Markers of the New Millennium. AACC Press, New York, pp115-117, 2002.

9. Al-Madhoun A, Tjarks W and Eriksson S: The role of thymidine kinases in the activation of pyrimidine nucleoside analogues. Mini Rev Med Chem 4: 341-350, 2004.

10. Jirawatnotai S,Hu Y, Michowski W, Elias JE, Becks L, Bienvenu F, Zagozdzon A, Goswami T, Wang YE, Clark AB, et al: A function for cyclin D1 in DNA repair uncovered by protein interactome analyses in human cancers. Nature 474: 230-234, 2011.

11. Cigna: Markers for diagnosis and management of cancer. USA, http://www.cigna.com. Accessed November 15, 2011.

12. Munch-Petersen B: Enzymatic regulation of cytosolic thymidine kinase 1 and mitochondrial thymidine kinase 2: a mini review. Nucleosides Nucleotides Nucleic Acids 29: 363-369, 2010.

13. Chen YL, Eriksson S and Chang ZF: Regulation and functional contribution of thymidine kinase 1 in repair of DNA damage. J Biol Chem 285: 27327-27335, 2010.

14. Gasparri F, Wang N, Skog S, Galvani A and Eriksson S: Thymidine kinase 1 expression defines an activated G1 state of the cell cycle as revealed with site-specific antibodies and ArrayScan assays. Eur J Cell Biol 88: 779-785, 2009.

15. He Q, Zou L, Zhang PA, Lui JX, Skog S and Fornander T: The clinical significance of thymidine kinase 1 measurements in serum of breast cancer patients using anti-TK1 antibody. Int J Biol Markers 15: 139-146, 2000.

16. Zou L, Zhang PG, Zou S, Li Y and He Q: The half-life of cytosolic thymidine kinase in serum by ECL dot bolt potential marker for monitoring the response to surgery of patients with gastric cancer. Int J Biolog Markers 17: 135-140, 2002.

17. Li HX, Zhang S, Lei DS, Wang XQ, Skog S and He Q: Serum thymidine kinase 1 is a prognostic and monitoring factor in patients with non-small cell lung cancer. Oncol Rep 13: 145-149, 2005

18. He Q, Zhang P, Zou L, Li H, Wang X, Zhou S, Fornander T and Skog S: Concentration of thymidine kinase 1 in serum (S-TK1) is a more sensitive proliferation marker in human solid tumors than its activity. Oncol Rep 14: 1013-1019, 2005.

19. Zhang J, Jia Q, Zou S, Zhang P, Zhang X, Skog S, Luo P, Zhang W and He Q: Thymidine kinase 1: A proliferation marker for determining prognosis and monitoring the surgical outcome of primary bladder carcinoma patients. Oncol Rep 15: 455-461, 2006.

20. He Q, Fornander T, Johansson H, Johansson U, Hu GZ, Rutqvist LE and Skog S: Thymidine kinase 1 in serum predicts increased risk of distant or loco-regional recurrence following surgery in patients with early breast cancer. Anticancer Res 26: 4753-4759, 2006.

21. Xu XH, Zhang YM, Shu XH, Shan LH, Wang ZW, Zhou YL, Wen HK, He F, He E and Skog S: Serological thymidine kinase 1 reflects progression of pre-malignant and malignant tumours during therapy. Mol Med Rep 1: 705-712, 2008.

22. Zhang GQ, Jiao SC and Wei XF: Serum thymidine kinase 1: a proliferation marker for neoplasms in oncologic monitoring of elderly people. J Modern Oncol 16: 831-832, 2008 (In Chinese)

23. Chen ZH, Zhou H, Tian NB, et al: Serological thymidine kinase (STK1) indicates an elevated risk for development of malignant tumours. Anticancer Res 28: 3897-3907, 2008.

24. Zhang XH, Yang JR, Fu XY, Liu HX, Yun X, Song B, Zhang M, $\mathrm{He} \mathrm{E}$ and Skog S: The significance of serum thymidine kinase 1 for the risk screening of cancer development in pre-cancerous diseases. Chin J Health Manag 4: 35-38, 2010.

25. Huang SQ, Lin J, Guo N, Zhang M, Yun X, Liu S, Zhou J, He E and Skog S: Elevated serum thymidine kinase 1 predicts risk of pre/early cancerous progression. Asian Pac J Cancer Prev 12: 497-505, 2011.

26. Chen ZH, Huang SQ, Wang Y, Yang AZ, Wen J, Xu XH, Chen Y Chen QB, Wang YH, He E, Zhou J and Skog S: Serological thymidine kinase 1 is a biomarker for early detection of tumours - a health screening study on 35,365 people, using a sensitive chemiluminescent dot blot assay. Sensors (Basel) 11: 11064-11080, 2011.

27. Chen ZH, Wang YQ, Zhu XL, Zhou H, Liu Y, Yang PT and Cao X: Thymidine kinase 1 measurement in health check up. Chin J Health Manag 45: 349-351, 2011.

28. Yuan ZY, Liu XA, Ling LJ, Zhao Y and Wang S: The significance of serum thymidine kinase 1 on assistant diagnosis and evaluation of chemotherapy effect on breast cancer patients operated. Acta Univ Med Nanjiang (Natural Science) 30: 1002-1004, 2010.
29. Huang $\mathrm{H}$ and Luo YL: The role of serum thymidine kinase 1 levels in the postoperative evaluation of curative effect of breast cancer. Lab Med 26: 79-81, 2011 (In Chinese).

30. Chen QB, Li CC, Zhao R, Dia Y, Chen QJ and Chen WY: The application study on serum thymidine kinase 1 level changes for diagnosis and therapy of breast cancers. Appl Med 28: 39-41, 2012.

31. Carlsson L, Larsson A and Lindman H: Elevated levels of thymidine kinase 1 peptide in serum from patients with breast cancer. Ups J Med Sci 114: 116-120, 2009.

32. Chen FY, Tang LL and Huang X: STK1 assay in patients with breast cancer before and after neo-adjuvant chemotherapy. Chin Tumor Clin 6: 732-735, 2009.

33. Lu J, Chen KM, Wang ZM, Wu CY and Zhou QH: Correlativity study on CT and serum thymidine kinase 1 of breast diseases Chin J CT MNR 7: 22-24, 2009.

34. Zhang ZY, Chen M,Fan L and Dong RF: Serum TK1 concentration and clinical significance in patients with cervical carcinoma. Jilin Med 32: 7438-7439, 2012.

35. Cheng XW, Yang YX, Yu XY and Liu YP: Expression of SCC-Ag and TK1 in middle-advanced cervical carcinoma and the significance in treatment. Chin J New Drugs 20: 1208-1300, 2011.

36. Chen Y, Ying M, Chen Y, Hu M, Lin Y, Chen D, Li X, Zhang M, Yun X, Zhou J, He E and Skog S: Serum thymidine kinase 1 correlates to clinical stages and clinical reactions and monitors the outcome of therapy of 1,247 cancer patients in routine clinical settings. Int J Clin Oncol 15: 359-368, 2010.

37. Li Z, Wang Y, He J, Ma J, Zhao L, Chen H, Li N, Zhou J, He E and Skog S: Serological thymidine kinase 1 is a prognostic factor in esophagus, cardiac and lung carcinomas. Eur J Cancer Prev 9: $313-318,2010$

38. Guo L and Lu RQ: Significance of serum thymidine kinase determination in patients with gastric carcinoma. Lab Med 23: 271-273, 2008

39. Xu LB, Shen LQ and Zhuang ZX: The expression and clinical significance of serumTK-1 in gastrointestinal tumors. J Clin Med Prac 17: 20-23, 2011 (In Chinese).

40. Shen Z, Chen Z, Xu R, Zhang J and Jiang H: Evaluation of the significance of serum thymidine kinase 1 detection in the diagnosis and curative effect of gastrointestinal malignant tumors. Exp Lab Med 5: 531-533, 2011 (In Chinese).

41. Jiao JH, Huan X and Shi RH: Research of thymidine kinase1(TK1) in diagnosis and follow-up of colorectal carcinoma. J Clin Med Prac 15: 21-23, 2011.

42. Xing $\mathrm{H}$ and Zhang ZF: Expression and clinical significance of serum TK1, OPN and CEA in patients with colorectal carcinoma. J Community Med 9: 15-18, 2011.

43. Tian JG, Jia YD and Liu HT: Serum TK1, EGF, HGF and CRP and IL-6 levels in patients with colon cancer using laparoscopic and open surgery. Shangdong Med 50: 42-43, 2011.

44. Zhang YC: Clinical significance of serum thymidine kinase 1 with colorectal carcinomas. J Med Forum 130: 28-30, 2009.

45. Wei JW, Xu CR, Zen DZ and Chen Y: Analysis on the content of TK1 of patients with colonic polyps. Lab Med Clin 8: 769-701, 2011.

46. Li SJ, Wu G, Ye J, Zhang X, Cheng F and Zhang KQ: The meaning of blood serum thymidine kinase level in early screening for prostatic carcinoma. Immun J 27: 459-461, 2011.

47. Luo P, He E, Eriksson S, Zhou J, Hu G, Zhang J and Skog S: Thymidine kinase activity in serum of renal cell carcinoma patients is a useful prognostic marker. Eur J Cancer Prev 18: 220-224, 2009.

48. Yuan H, Zhu B, Wu YW and Zhou JT: Clinical significance of determination of changes of serum TK1 level in patients with lung cancer. J Radioimmunol 24: 301-302, 2011 (In Chinese).

49. Zhou Y, Wang D, Yang AZ, He E, Skog S and Zhang M: Clinical significant of detecting serum thymidine kinase 1 in diagnostic and prognosis evaluation of lung cancers. Jiangshu Med 38: 310-313, 2012

50. Guan Y, Guo QS, Shan FL and Liu IJ: Expression and significance of thymidine kinase 1 in small cell lung carcinoma tissues. Chin J Cancer Prev Treat 17: 746-748, 2010.

51. Feng MH: The significance of the detection of serum thymidine kinase in treatment assessment of lung carcinomas. J Radioimmunol 24: 455-456, 2011.

52. $\mathrm{Xu} \mathrm{W}$, Shen QD and Yu H: Serum thymidine kinase and LPL and its associated prognostic factors in chronic lymphocytic leukemia. Chin J Hematol 30: 23-26, 2009.

53. Xu W, Cao X, Miao KR, Qiao C, Wu YJ, Liu Q, Fan L and Li JY: Serum thymidine kinase 1 concentration in Chinese patients with chronic lymphocytic leukemia and its correlation with other prognostic factors. Int J Hematol 90: 205-211, 2009. 
54. Zeng ZJ, Uyang WT, Sun YH and Cai XH: Clinical significance of thymidine kinase 1 in chemotherapy of leukemia. J Mol Diagn Ther 1: 28-31, 2009.

55. Pan ZL, Ji XY, Shi YM, Zhou J, He E and Skog S: Serum thymidine kinase 1 concentration as a prognostic factor of chemotherapy-treated non-Hodgkin's lymphoma patients. J Cancer Res Clin Oncol 136: 1193-1199, 2010.

56. Chen QB, Liu RP, Huang H, Li Q, Zhang JY, Zhen SB, Li Q, Jing N and Zhen L: Serum thymidine kinase 1 and its role in monitoring and prognosis of tumor. Guangdong Med 29: 1022-1024, 2008.

57. Li QR, Yuan H, Sang SB, Su CH, Wu YW, Zhang M, Zhou J, Zhao JT, He E and Skog S: The significance of serum thymidine kinase 1 for early tumor detection and monitoring the response to therapy. Chin J Nuclear Med 29: 271-273, 2009.

58. Wang LJ: Clinical significance of cytoplasmic thymidine kinase in patients with malignant tumors. Heilongjiang Med 24: 904-905, 2011.

59. Wang ZX, Zhang B, Ni B and Zhan J: Value of serum thymidine kinase 1 in evaluating the efficacy of malignant tumor treatment. Jiangsu Med J 18: 2174-2175, 2011 (In Chinese).

60. Mao RY, Wu JP, Wang N, He LX, Wu CJ, He Q and Skog S: A comparison study: immunohistochemical detection of cytosolic thymidine kinase and proliferating cell nuclear antigen in breast cancer. Cancer Invest 20: 922-931, 2002.

61. Wu JP, Mao YR, He LX, Wang N, Wu CJ, He Q and Skog S: Comparison study on the expression of cytosolic thymidine kinase and proliferating cell nuclear antigen in colorectal carcinoma. Anticancer Res 20: 4815-4820, 2000.

62. He Q, Mao Y, Wu J, Decker C, Merza M, Wang N, Eriksson S, Castro $\mathrm{J}$ and Skog S: Cytosolic thymidine kinase is a specific histopathologic tumour marker for breast carcinomas. Int J Oncol 25: 945-953, 2004.

63. Mao YR, Wu JP, Skog S, Eriksson S, Zhao Y, Zhou J and He Q: Expression of cell proliferating genes in patients with non-smallcell lung cancer (NSCLC) by immunohistochemistry and cDNA profiling. Oncol Rep 13: 837-846, 2005.

64. Guan H, He E, Zhou J, Li Y, Eriksson S and Skog S: Thymidine kinase 1 expression is significantly different in breast benign (UDH), and in pre-malignant (ADH), primary tumour in situ (DCIS) and invasive ductal (IDC) of breast carcinoma. Mol Med Rep 2: 923-929, 2009

65. Liu C, Gao Q, Shi QL, Yu B, Ma HH, Eriksson S, He E and Skog S: Significance of TK1 and Ki-67 expression in ovarian serous adenocarcinoma. J Clin Exp Pathol 27: 1289-1293, 2011.

66. Xu Y, Shi QL, Ma H, Zhou H, Lu Z, Yu B, Zhou X, Eriksson S, $\mathrm{He} \mathrm{E}$ and Skog S: High thymidine kinase 1 (TK1) expression is a predictor of poor survival in patients with pT1 of lung adenocarcinoma. Tumour Biol 33: 475-483, 2012.

67. Luo P, Wang N, He E, Eriksson S, Zhou J, Hu G, Zhang J and Skog S: The proliferation marker thymidine kinase 1 level is high in normal kidney tubule cells compared to other normal and malignant renal cells. Pathol Oncol Res 16: 277-283, 2010.
68. Gakis G, Hennenlotter J, Scharpf M, Hevler J, Schilling D, Kuehs U, Stenzl A and Schwentner C: XPA-210: a new proliferation marker to characterize tumor biology and progression of renal cell carcinoma. World J Urol 29: 801-806, 2011.

69. Ye FP, Xie QL, Liu XL, Zhen JH, Lan JL and Yu M: Expression of TK1 and Ki67 in prostate diseases. J Clin Exp Pathol 24: 644-667, 2008.

70. Aufderklamm S, Hennenlotter J, Todenhoefer T, Gakis G, Schilling D, Vogel U, Kuehs U, Dlugosch J, Knapp J, Merseburger A, et al: XPA-210: a new proliferation marker determines locally advanced prostate cancer and is a predictor of biochemical recurrence. World J Urol: Oct 4, 2011 (Epub ahead of print).

71. Yang L, Parkin DM, Ferlay J, Li L and Chen Y: Estimates of cancer incidence in China for 2000 and projections for 2005. Cancer Epidemiol Biomarkers Prev 14: 243-250, 2005.

72. Barregard L, Holmberg E and Sallsten G: Leukaemia incidence in individualsliving close to an oil refinery. Environ Res 109: 985-990, 2009

73. Berman JJ and Moor GW: Precancer: The Beginning and the End of Cancer. Jones and Bartlett Learning Inc., Boston, 2010.

74. Nisman B, Allweis T, Kaduri L, Maly B, Gronowitz S, Hamburger T and Peretz T: Serum thymidine kinase 1 activity in breast cancer. Cancer Biomark 7: 65-72, 2010.

75. Kemik O, Kemik AS, Purisa S and Tuzun S: Serum thymidine kinase is associated with gastric adenocarcinoma. Bratisl Lek Listy 112: 510-511, 2011.

76. Letocha H, Eklov S, Gronowitz S, Johan BN and Nilsson S: Deoxythymidine kinase in the staging of prostatic adenocarcinoma. Prostate 29: 15-19, 1996.

77. Nisman B, Yutkin V, Nechushtan H, Gofrit ON, Peretz T, Gronowitz S and Pode D: Circulating tumor M2 pyruvate kinase and thymidine kinase 1 are potential predictors for disease recurrence in renal cell carcinoma after nephrectomy. Urology 76: 513-516, 2010.

78. Konoplev SN, Fritsche HA, O'Brien S, Wierda WG, Keating MJ, Gornet TG, St Romain S, Wang X, Inamdar K, Johnson MR, et al: High serum thymidine kinase 1 level predicts poorer survival in patients with chronic lymphocytic leukemia. Am J Clin Pathol 134: 472-477, 2010.

79. Procházka V, Faber E, Raida L, Langová K, Indrák K and Papajík T: High baseline serumthymidine kinase 1 level predicts unfavorable outcome in patients with follicular lymphoma. Leuk Lymphoma 53: 1306-1310, 2012. 